\title{
Cadmium affects the regeneration of the leafy vegetable Talinum portulacifolium stem cuttings in nutrient solution
}

Thangavelu Muthukumar \& Selvam Dinesh-Babu

Root and Soil Biology Laboratory, Department of Botany, Bharathiar University, Coimbatore - 641046, Tamilnadu, India.

Correspondence

T. Muthukumar

E-mail: tmkum@yahoo.com

Received: 13 February 2020

Accepted: 20 July 2020

Published on-line: 4 December 2020

\section{Resumen}

El cadmio afecta a la regeneración de los esquejes en solución nutritiva de la verdura de hoja Talinum portulacifolium

Investigamos el efecto de varias concentraciones (0,0-5,0 ppm) de cadmio (Cd) en la capacidad de regeneración; las características morfológicas y la acumulación de $\mathrm{Cd}$ en los esquejes de tallo de la verdura de hoja Talinum portulacifolium cultivada en cultivo hidropónico. El Cd retrasó la brotación de los esquejes en un 7\%, la callosidad en un $8 \%$ y el enraizamiento en un $38 \%$. Las diferentes concentraciones de $\mathrm{Cd}$ afectaron significativamente a los pesos fresco y seco de las partes de la planta, excepto las raíces. La acumulación de Cd fue mayor en los tallos que en las hojas $(2,22$ vs $0,57 \mathrm{ppm}$ ). El índice de tolerancia calculado osciló entre el $59 \%$ y el $88 \%$. Basándose en las observaciones, se concluyó que el Cd interfiere con la regeneración de los esquejes de tallo de T. portulacifolium e implica preocupación sobre el consumo y el uso terapéutico de esta hortaliza de hoja que crece en suelos contaminados.

Palabras clave: Callo; Crecimiento; Metales pesados; Hidropónico; Enraizamineto; Índice de tolerancia.

\begin{abstract}
We investigated the effect of various concentrations $(0.0-5.0 \mathrm{ppm})$ of cadmium ( $\mathrm{Cd}$ ) on the regeneration ability; morphological characteristics and $\mathrm{Cd}$ accumulation in the leafy vegetable Talinum portulacifolium stem cuttings grown in hydroponic culture. Cd delayed sprouting of stem cuttings by $7 \%$, callusing by $8 \%$ and rooting by $38 \%$. Different $\mathrm{Cd}$ concentrations significantly affected fresh and dry weight of plant parts except roots. Accumulation of $\mathrm{Cd}$ was more in the stems than in leaves (2.22 vs $0.57 \mathrm{ppm})$. The calculated tolerance index ranged from $59 \%$ to $88 \%$. Based on the observations it was concluded that $\mathrm{Cd}$ interferes with the regeneration of $T$. portulacifolium stem cuttings and imply concerns on the consumption and therapeutic use of this leafy vegetable growing on polluted soils.
\end{abstract}

Key words: Callus; Growth; Heavy Metals; Hydroponics; Rooting; Tolerance index. 


\section{Introduction}

Pollution of natural resources like water and soil by heavy metals (HMs) is a major concern for plant and human health because of their longtime persistent effect in the environment. HMs is one of the most dangerous environmental pollutants as most of these HMs are toxic even at very low concentrations. Pollution of the environment by toxic HMs has undisputedly become an issue of worldwide concern after industrialization due to its toxic and long lasting effects (Friberg et al. 2019). Humans are directly exposed to HMs due to the consumption of crops and vegetables grown on HM contaminated soils and even ground water are contaminated with HMs (Jackson \& Alloway 2017).

Among the various HMs that pollute the environment cadmium $(\mathrm{Cd})$ with no known biological function is toxic for both plants and humans (Jackson \& Alloway 2017, Shahid et al. 2016). Low concentrations of $\mathrm{Cd}$ occurs naturally in the soil and $\mathrm{Cd}$ originating from metal industry, plastics, sewage and phosphate fertilizers increases the presence of this HM in the soil (Shahid et al. 2016). The high mobility of $\mathrm{Cd}$ in the soil renders it 2 to 20 times more toxic than other HMs (Friberg et al. 2019, Khan et al. 2017). Even at low concentrations, $\mathrm{Cd}$ can negatively affect plant growth, metabolism of sugars, assimilation of sulphate and activities of different enzymes (Shahid et al. 2016) and cellular activities like cell proliferation and their differentiation in humans and animals (He et al. 2017, Waisberg 2003). In India, soils and ground water affected by various anthropogenic activities have $\mathrm{Cd}$ concentrations of 12.8$90.0 \mathrm{mg} / \mathrm{kg}$ and $40-280 \mu \mathrm{g} / \mathrm{L}$ respectively (Kubier et al. 2019).

De-contamination of $\mathrm{Cd}$ from soils is receiving increasing attention from the public as well as governmental bodies, particularly in developing countries (Khan et al. 2017). Plant based technologies are applicable for removing HMs from areas of low concentrations with shallow soils and water, although longer treatment time may be required (Clemens et al. 2013). However, when using a plant based system it is important to understand the toxic effects of $\mathrm{Cd}$ on the plants and also the ability of plants to grow or regenerate in such toxic environments.

Hydroponics not only reduces the period of plant growth and the duration of the experiments but also lowers the space required for carrying the experiment. Other advantages of hydroponics include maneuverability due to the distinct characteristics of the liquid medium, easy observation of the intact root system and undisturbed monitoring of changes in the root zones (Zhi-xin et al. 2007). The bioavailability of an HM in the soil is mainly affected by the total content of other HMs in the soil, chemical and physical properties of the soil and also the plant species (Shahid et al. 2016). So in order to get a clear understanding of the effects of a particular HM, researchers have focused their attention on the hydroponic system (McBride et al. 2016, Wang et al. 2016). Hydroponics help to understand the direct and exact effect of $\mathrm{Cd}$ or other HMs on plants as there is no interference from other soil factors. Moreover, we will also have an idea on the ability of plants in absorbing, concentrating or precipitating toxic metals from polluted effluents (Dushenkov et al. 1995).

Talinum Adans, belonging to the plant family Portulaceae, consists of herbaceous succulent plants. The leaves of several Talinum species are edible and are widely cultivated in tropical regions. The genus Talinum consists of 15 species of which five occur in India (Swarna et al. 2015).

Talinum species like Talinum fruticosum (L.) Juss. (Adefemi et al. 2012, Babyemi et al. 2017, Ebong et al. 2018, Kumar \& Prasad 2015), Talinum paniculatum (Jacq.) Gaertn. (de Souza et al. 2018) and Talinum portulacifolium (Forssk.) Asch. ex Schweinf. (Sekhar et al. 2007) were examined for their ability to accumulate or tolerate different HMs.

In the present study, the model plant $T$. portulacifolium Asch. ex Schweinf., is an erect undershrub and the leaves of this plant are cooked and consumed as a vegetable or used raw in salads (Jansen 2004, Nair \& Henry 1983). Moreover, the leaves of this plant are also used for treating eye diseases; roots are used for curing cough and gonorrhea and the whole plant is believed to possess aphrodisiac properties and relieve constipation (Jansen 2004). In India, T. portulacifolium is found in Assam, Western and North-Western India, and Peninsular India (Nair \& Henry 1983, Sankara Rao et al. 2019). Further T. portulacifolium is popularly propagated through stem cuttings for cultivation in home gardens and 
Kumar and Prasad (2010) suggested that this plant species in hydroponic media could serve as a tool to study various aspects of environmental pollution. As a multiutility plant, the influence of $\mathrm{Cd}$ on the regeneration of $T$. portulacifolium and its efficacy to accumulate the heavy metal would enable us to understand the risks associated with the collection and consumption of this leafy vegetable from $\mathrm{Cd}$ contaminated/rich soils. Therefore, in this study, we focused on the effect of different concentrations of $\mathrm{Cd}$ on the regeneration and morphological characteristics of $T$. portulacifolium. In addition, the ability of $T$. portulacifolium to accumulate $\mathrm{Cd}$ from the hydroponic solution without the interference of other soil factors was also examined.

\section{Material and methods}

\section{Plant material and experimental design}

Herbaceous stem cuttings ( $\sim 120 \mathrm{~mm}$ long and $\sim 10$ $\mathrm{mm}$ thick) of $T$. portulacifolium were collected from plants growing in pots containing uncontaminated Alfisol soil at Bharathiar University Campus $\left(11^{\circ} 2^{\prime} 20.4792^{\prime \prime N}, 76^{\circ} 52^{\prime} 35.1084^{\prime \prime E}\right)$, Coimbatore, India. The unrooted stem cuttings $(120 \mathrm{~mm}$ long and $10 \mathrm{~mm}$ thick) were prepared using a sharp stainless steel knife. The two-way factorial experiment consisted of 11 concentrations of $\mathrm{Cd}$ and five growth periods with five replicates. The observations for growth periods one to four were non-destructive and there were $55(11 \times 5)$ experimental units. The cuttings were placed in glass containers filled with full-strength Hoagland nutrient solution $\left(\mathrm{KNO}_{3}, \mathrm{Ca}\left(\mathrm{NO}_{3}\right)_{2} \cdot 4 \mathrm{H}_{2} \mathrm{O}, \mathrm{MgSO}_{4}\right.$ $\cdot 7 \mathrm{H}_{2} \mathrm{O}, \mathrm{NH}_{4} \mathrm{H}_{2} \mathrm{PO}_{4}, \mathrm{MnCl}_{2} \cdot 4 \mathrm{H}_{2} \mathrm{O}, \mathrm{H}_{3} \mathrm{BO}_{3}, \mathrm{MoO}_{3}$, $\mathrm{ZnSO}_{4} \cdot 7 \mathrm{H}_{2} \mathrm{O}, \mathrm{CuSO}_{4} \cdot 5 \mathrm{H}_{2} \mathrm{O}, \mathrm{FeSO}_{4} \cdot 7 \mathrm{H}_{2} \mathrm{O}$ ) (Hoagland and Arnon 1950), that was either unspiked or spiked with a known concentration of Cd. Two stem cuttings were placed in each glass container containing $150 \mathrm{~mL}$ of the $\mathrm{Cd}$ unspiked or spiked nutrient solution. The glass containers were covered with aluminium foil to prevent the nutrient solution from exposure to light. The cuttings were placed in such a way that only the lower part of the twig (approximately one to two $\mathrm{cm}$ ) was immersed in the metal solution. The solutions were replaced every $7^{\text {th }}$ day throughout the experiment. The cuttings were placed at room temperature (28 $\pm 2{ }^{\circ} \mathrm{C}$ ) with $14 \mathrm{~h}$ day $/ 10 \mathrm{~h}$ dark period at a light intensity of 300 to $350 \mu \mathrm{mol} / \mathrm{m}^{2} \mathrm{~s}$.

\section{Preparation of the Cd solution}

The concentrations of $\mathrm{Cd}$ used in the present study was based on a trial run where stem cuttings of T. portulacifolium were grown in a wide range of $\mathrm{Cd}$ spiked nutrient solutions $(0,5,10,15$ and $20 \mathrm{ppm}$ ) for 20 days. As the stem cuttings raised in Cd concentrations beyond $5.0 \mathrm{ppm}$ failed to develop and even cuttings raised in this $\mathrm{Cd}$ concentration exhibited toxicity symptoms like browning and reduced regeneration. Hence the Cd concentrations for the present study were fixed between 0 and $5.0 \mathrm{ppm}$. Cadmium chloride $\left(\mathrm{CdCl}_{2}\right)$ formed the sources of $\mathrm{Cd}$ and the solution containing $\mathrm{Cd}$ was prepared by dissolving known quantities of $\mathrm{CdCl}_{2}$ in Hoagland nutrient solution. Required concentrations $(0.0,0.5,1.0,1.5,2.0,2.5,3.0,3.5$, 4.0, 4.5, $5.0 \mathrm{ppm}$ ) of Cd was prepared individually by diluting the stock solution (1000 ppm; $2.036 \mathrm{~g}$ of $\mathrm{CdCl}_{2}$ in $1 \mathrm{~L}$ ) using Hoagland nutrient solutions. The treatments are hereafter coded as $\mathrm{Cd} 0.0$, Cd0.5, Cd1.0, Cd1.5, Cd2.0, Cd2.5, Cd3.0, Cd3.5, Cd4.0, Cd4.5 and Cd5.0. Hoagland nutrient solution without $\mathrm{CdCl}_{2}(0.0 \mathrm{ppm})$ served as the medium for growth of control plants.

\section{Plant growth measurements and harvest}

The duration (in days) for sprouting, root initiation and callus formation after exposure to $\mathrm{Cd}$ solutions were observed visually. The number of shoots, shoot length, leaf number, root number and total root length (length of all individual roots) was measured non-destructively at 7, 14, 21 and 28 (termed as D7, D14, D21 and D28) and destructively at 35 days (D35) after the initiation of the experiment. The cuttings were finally harvested destructively at D35, washed in distilled water and the leaves, stems and roots were separated. After recording the fresh weights, the plant parts were dried at $78{ }^{\circ} \mathrm{C}$ for 48 hours in a hot air oven to record their dry weight. The magnitude of stem decay and toxicity symptoms was assessed visually.

\section{Heavy metal analysis in cuttings}

Dried plant samples (leaves and stem) were ground into a fine powder and sieved through a $0.2 \mathrm{~mm}$ sieve. The plant samples were wet acid digested with $\mathrm{HNO}_{3}, \mathrm{H}_{2} \mathrm{SO}_{4}$, and $\mathrm{HClO}_{4}$ in the ratio of 9:2:1 (Antosiewicz 1993, Piper 1966) for quantifying their total $\mathrm{Cd}$. All the plant digests were analyzed for $\mathrm{Cd}$ concentration using Atomic 
Absorption Spectrophotometer (Varian Techtran Spectr AA 10/20 BQ, Australia). Due care was taken to avoid metal contamination during the entire process of harvesting, washing, drying and grinding. Cd analysis could not be performed for roots due to inadequate sample in certain treatments.

\section{Tolerance index (Ti)}

The Ti was calculated to measure the ability of the plant to grow in the presence of a given concentration of metal, according to Wilkins (1978) using the formula:

$\mathrm{Ti}=[$ Dry weight of plants grown in Cd solution / Dry weight of plants grown in Cd free solution] $\times 100$

\section{Statistical analysis}

Mean values of morphological growth parameters of plants were calculated from five replicates in each concentration. Analysis of Variance (oneway or two-way) was performed after analyzing the data for normality (Levene's test). When the F values were significant, Duncan's Multiple Range Test (DMRT) was used to compare the variation among treatments. Pearson's correlation or regression analysis was used to assess the relation of $\mathrm{Cd}$ concentration to different regeneration, growth and $\mathrm{Cd}$ accumulation parameters. All statistical analysis was performed using Statistical Package for Social Science (SPSS, Version 9.0).

\section{Results}

\section{Effect of $\mathrm{Cd}$ on the regeneration of $T$. portulacifolium}

Stem cuttings sprouted between D2 and D3 after their exposure to different concentrations of $\mathrm{Cd}$, whereas, root initiation was delayed up to the D12 at the highest concentration Cd5.0 (Fig. 1). Though the differences in time taken for sprouting in different concentrations of $\mathrm{Cd}$ were not significant $\left(\mathrm{F}_{8,36}=<1 ; \mathrm{p}>0.05\right)$, the differences in duration for root initiation $\left(\mathrm{F}_{8,36}=6.84 ; \mathrm{p}<0.01\right)$ and callus formation $\left(\mathrm{F}_{8,36}=3.12 ; \mathrm{p}<0.05\right)$ were significant. As concentrations of $\mathrm{Cd}$ was significantly and positively correlated to days for root initiation $(\mathrm{r}=0.898 ; \mathrm{p}<0.001 ; \mathrm{n}=11)$, no such correlation ( $\mathrm{p}>0.05 ; \mathrm{n}=11$ ) was found for days taken for sprouting $(\mathrm{r}=0.492)$ or callus formation $(\mathrm{r}=0.250)$ (Fig. 1).

Different concentrations of $\mathrm{Cd}$ and growth

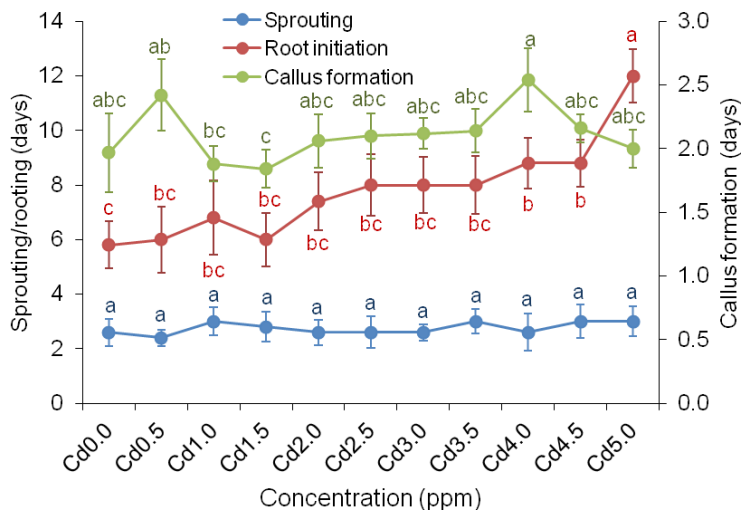

Figura 1. Influencia de diferentes concentraciones de cadmio (Cd) en la formación del callo, germinación y enraizamiento de esquejes de Talinum portulacifolium en cultivo hidropónico. Las barras de error indican error estándar. Puntos de una variable con la misma(s) letra(s) no son significativamente diferentes $(\mathrm{p}>0,05)$ según la prueba de rango múltiple de Duncan.

Figure 1. Influence of different concentrations of cadmium $(\mathrm{Cd})$ on callus formation, sprouting and rooting of Talinum portulacifolium stem cuttings in hydroponic culture. Error bars indicate \pm standard error. Points for a variable bearing same letter(s) are not significantly different $(p>0.05)$ according to Duncan's Multiple Range Test

period failed to significantly influence the number of shoot apices (Table 1). The two-way interaction for the main factors (day $\times$ treatment) was also not significant for shoot apices. Shoot lengths and leaf numbers of $T$. portulacifolium stem cuttings significantly varied with growth period and were significantly influenced by the concentrations of $\mathrm{Cd}$. However, the interaction day $\times$ treatment was not significant for these variables. Root numbers and total root length significantly varied with growth period and was significantly influenced by different concentrations of $\mathrm{Cd}$ in the nutrient solution. Though the interaction day $\times$ treatment was significant for total root length it was insignificant for root numbers (Table 1).

The numbers of shoot apices were almost similar over a period of 35 days and were not influenced by the increasing concentrations of $\mathrm{Cd}$. On D7, maximum shoot length was observed in $\mathrm{Cd} 0.0$ and there was a $72 \%$ decrease in $\mathrm{Cd} 4.5$ which recorded the minimum shoot length. As the number of roots was maximum in $\mathrm{Cd} 0.5$, roots were absent in $\mathrm{Cd} 3.0-\mathrm{Cd} 5.0$. Moreover, root elongation was inhibited by different concentrations of $\mathrm{Cd}$, maximum root length was observed in $\mathrm{Cd} 0.5$. Different concentrations of $\mathrm{Cd}$ was significantly and negatively correlated to shoot length, root numbers and total root length at the D7 (Table 2).

On D14, shoot length ranged between $3.78 \mathrm{~cm}$ $(\mathrm{Cd} 0.0)$ and $1.9 \mathrm{~cm}(\mathrm{Cd} 4.5)$. More number of 


\begin{tabular}{|c|c|c|c|c|c|c|}
\hline Days(D) & $\begin{array}{c}\text { Cd concentration } \\
(\mathrm{ppm})\end{array}$ & $\begin{array}{l}\text { Shoot apex } \\
\text { (per cutting) }\end{array}$ & $\begin{array}{l}\text { Shoot length } \\
(\mathrm{cm})\end{array}$ & $\begin{array}{l}\text { Leaf number } \\
\text { (per cutting) }\end{array}$ & $\begin{array}{l}\text { Root number } \\
\text { (per cutting) }\end{array}$ & $\begin{array}{l}\text { Total root } \\
\text { length }(\mathrm{cm})\end{array}$ \\
\hline & Cd 0.0 & $2.8 \pm 0.37 a$ & $1.16 \pm 0.08 a$ & $10.6 \pm 0.75 a$ & $19.6 \pm 0.81 c$ & $0.64 \pm 0.05 b$ \\
\hline & $\mathrm{Cd} 0.5$ & $3.0 \pm 0.45 a$ & $0.90 \pm 0.13 b$ & $9.0 \pm 0.71 \mathrm{abc}$ & $27.6 \pm 0.93 a$ & $1.58 \pm 0.11 \mathrm{a}$ \\
\hline & Cd 1.0 & $2.2 \pm 0.37 \mathrm{a}$ & $0.72 \pm 0.04 b c$ & $5.2 \pm 0.37 \mathrm{~d}$ & $2.0 \pm 0.71 \mathrm{e}$ & $0.18 \pm 0.02 \mathrm{~cd}$ \\
\hline & Cd 1.5 & $2.6 \pm 0.24 a$ & $0.92 \pm 0.07 b$ & $9.8 \pm 0.86 a b$ & $22.4 \pm 1.21 \mathrm{~b}$ & $0.26 \pm 0.02 c$ \\
\hline & $\mathrm{Cd} 2.0$ & $2.6 \pm 0.24 a$ & $0.68 \pm 0.07 c$ & $7.8 \pm 0.58 b c$ & $7.6 \pm 1.12 \mathrm{~d}$ & $0.14 \pm 0.02 d$ \\
\hline \multirow[t]{11}{*}{ D7 } & $\mathrm{Cd} 2.5$ & $2.8 \pm 0.20 a$ & $0.68 \pm 0.06 c$ & $8.6 \pm 0.51 \mathrm{abc}$ & $6.0 \pm 0.71 \mathrm{~d}$ & $0.10 \pm 0.03$ ef \\
\hline & Cd 3.0 & $2.8 \pm 0.20 a$ & $0.68 \pm 0.04 c$ & $9.4 \pm 0.87 \mathrm{abc}$ & $0.0 \pm 0.00 \mathrm{f}$ & $0.00 \pm 0.00 \mathrm{f}$ \\
\hline & Cd 3.5 & $2.4 \pm 0.24 a$ & $0.60 \pm 0.04 \mathrm{c}$ & $7.8 \pm 0.58 \mathrm{bc}$ & $0.0 \pm 0.00 \mathrm{f}$ & $0.00 \pm 0.00 \mathrm{f}$ \\
\hline & $\mathrm{Cd} 4.0$ & $2.8 \pm 0.58 a$ & $0.74 \pm 0.05 b c$ & $10.4 \pm 0.87 a$ & $0.0 \pm 0.00 \mathrm{f}$ & $0.00 \pm 0.00 \mathrm{f}$ \\
\hline & $\mathrm{Cd} 4.5$ & $3.0 \pm 0.63 a$ & $0.32 \pm 0.07 \mathrm{~d}$ & $7.2 \pm 0.58 c$ & $0.0 \pm 0.00 \mathrm{f}$ & $0.00 \pm 0.00 f$ \\
\hline & Cd 5.0 & $2.6 \pm 0.24 \mathrm{a}$ & $0.56 \pm 0.05 c$ & $9.2 \pm 0.58 \mathrm{abc}$ & $0.0 \pm 0.00 \mathrm{f}$ & $0.00 \pm 0.00 \mathrm{f}$ \\
\hline & Cd 0.0 & $2.8 \pm 0.37 a$ & $3.78 \pm 0.24 a$ & $19.8 \pm 0.86 a$ & $34.6 \pm 1.81 \mathrm{a}$ & $0.80 \pm 0.07 \mathrm{~b}$ \\
\hline & Cd 0.5 & $3.0 \pm 0.32 \mathrm{a}$ & $3.46 \pm 0.22 a$ & $18.2 \pm 1.24 a b$ & $28.2 \pm 1.28 b$ & $0.50 \pm 0.07 \mathrm{cde}$ \\
\hline & Cd 1.0 & $2.2 \pm 0.49 a$ & $2.64 \pm 0.17 b c$ & $16.4 \pm 0.93 \mathrm{bcd}$ & $16.2 \pm 0.86 \mathrm{de}$ & $0.38 \pm 0.06 \mathrm{def}$ \\
\hline & Cd 1.5 & $2.6 \pm 0.40 a$ & $2.80 \pm 0.13 b$ & $16.0 \pm 1.22 \mathrm{bcd}$ & $21.6 \pm 0.93 c$ & $0.62 \pm 0.09 \mathrm{~cd}$ \\
\hline & $\mathrm{Cd} 2.0$ & $2.8 \pm 0.49 a$ & $2.76 \pm 0.21 b$ & $17.8 \pm 0.37 \mathrm{abc}$ & $19.2 \pm 1.07 \mathrm{~cd}$ & $0.48 \pm 0.06 \mathrm{cde}$ \\
\hline \multirow[t]{11}{*}{ D14 } & $\mathrm{Cd} 2.5$ & $3.0 \pm 0.32 a$ & $2.52 \pm 0.08 b c$ & $16.0 \pm 1.05 \mathrm{bcd}$ & $17.2 \pm 0.80 \mathrm{de}$ & $0.52 \pm 0.04 \mathrm{~cd}$ \\
\hline & Cd 3.0 & $2.8 \pm 0.49 a$ & $2.50 \pm 0.11 b c$ & $14.8 \pm 0.86 \mathrm{cde}$ & $17.8 \pm 1.07 \mathrm{de}$ & $0.30 \pm 0.07$ efg \\
\hline & Cd 3.5 & $2.4 \pm 0.24 \mathrm{a}$ & $2.50 \pm 0.15 b c$ & $12.6 \pm 0.93 \mathrm{e}$ & $17.8 \pm 1.24 \mathrm{de}$ & $0.14 \pm 0.02 \mathrm{~g}$ \\
\hline & Cd 4.0 & $2.8 \pm 0.37 a$ & $2.14 \pm 0.14 \mathrm{~cd}$ & $14.0 \pm 1.14 \mathrm{de}$ & $14.6 \pm 0.93 \mathrm{e}$ & $0.38 \pm 0.07$ def \\
\hline & Cd 4.5 & $2.8 \pm 0.37 a$ & $1.91 \pm 0.12 \mathrm{~d}$ & $16.0 \pm 0.63 \mathrm{bcd}$ & $16.2 \pm 0.97 \mathrm{de}$ & $1.08 \pm 0.11 a$ \\
\hline & Cd 5.0 & $2.6 \pm 0.24 a$ & $2.14 \pm 0.14 \mathrm{~cd}$ & $15.8 \pm 0.86 \mathrm{bcd}$ & $11.2 \pm 0.80 \mathrm{f}$ & $0.24 \pm 0.04 \mathrm{fg}$ \\
\hline & Cd0.0 & $2.8 \pm 0.20 a$ & $5.34 \pm 0.16 a$ & $21.4 \pm 0.60 \mathrm{a}$ & $48.4 \pm 1.08 \mathrm{a}$ & $1.62 \pm 0.07 \mathrm{a}$ \\
\hline & Cd 0.5 & $3.0 \pm 0.32 a$ & $5.56 \pm 0.11 \mathrm{a}$ & $20.0 \pm 1.14 a b$ & $36.4 \pm 0.93 b$ & $0.64 \pm 0.02 \mathrm{de}$ \\
\hline & Cd 1.0 & $2.2 \pm 0.49 a$ & $4.68 \pm 0.12 b$ & $15.8 \pm 0.37 \mathrm{e}$ & $20.8 \pm 0.86 f$ & $0.44 \pm 0.05$ ef \\
\hline & Cd 1.5 & $2.6 \pm 0.40 a$ & $4.82 \pm 0.14 b$ & $17.2 \pm 0.58 b-e$ & $31.2 \pm 0.97 c$ & $0.64 \pm 0.09 \mathrm{de}$ \\
\hline & $\mathrm{Cd} 2.0$ & $2.8 \pm 0.58 a$ & $5.22 \pm 0.14 a$ & $16.4 \pm 0.93 \mathrm{de}$ & $27.6 \pm 1.03 \mathrm{de}$ & $1.41 \pm 0.11 \mathrm{~b}$ \\
\hline \multirow[t]{11}{*}{ D21 } & Cd 2.5 & $2.8 \pm 0.20 a$ & $4.76 \pm 0.12 b$ & $20.6 \pm 0.81 a$ & $38.6 \pm 1.36 \mathrm{~b}$ & $0.64 \pm 0.05 \mathrm{de}$ \\
\hline & $\mathrm{Cd} 3.0$ & $2.8 \pm 0.58 a$ & $3.76 \pm 0.14 \mathrm{~cd}$ & $15.0 \pm 0.89 \mathrm{e}$ & $29.4 \pm 1.08 \mathrm{cde}$ & $0.40 \pm 0.09 f$ \\
\hline & Cd 3.5 & $2.6 \pm 0.68 \mathrm{a}$ & $3.58 \pm 0.12 \mathrm{~d}$ & $16.0 \pm 0.71 \mathrm{de}$ & $27.8 \pm 0.86 \mathrm{de}$ & $0.66 \pm 0.05 d$ \\
\hline & Cd 4.0 & $2.8 \pm 0.58 a$ & $4.06 \pm 0.11 \mathrm{c}$ & $16.8 \pm 1.07 \mathrm{cde}$ & $26.6 \pm 0.93 \mathrm{e}$ & $0.71 \pm 0.06 \mathrm{~d}$ \\
\hline & Cd 4.5 & $3.0 \pm 0.32 \mathrm{a}$ & $4.78 \pm 0.16 b$ & $19.4 \pm 1.08 \mathrm{abc}$ & $30.2 \pm 0.86 \mathrm{~cd}$ & $1.08 \pm 0.07 c$ \\
\hline & Cd 5.0 & $2.8 \pm 0.37 a$ & $3.16 \pm 0.11 \mathrm{e}$ & $18.8 \pm 1.36 \mathrm{a}-\mathrm{d}$ & $28.4 \pm 0.93 \mathrm{cde}$ & $0.51 \pm 0.05$ def \\
\hline & $\mathrm{Cd} 0.0$ & $2.8 \pm 0.37 a$ & $5.68 \pm 0.09$ be & $22.8 \pm 0.66 \mathrm{a}$ & $48.4 \pm 1.44 \mathrm{a}$ & $1.82 \pm 0.09 a$ \\
\hline & Cd 0.5 & $3.0 \pm 0.55 a$ & $6.38 \pm 0.25 a$ & $21.6 \pm 0.81 a$ & $39.4 \pm 1.25 b$ & $0.68 \pm 0.06 \mathrm{~cd}$ \\
\hline & Cd 1.0 & $2.2 \pm 0.37 a$ & $5.28 \pm 0.14 d$ & $16.8 \pm 1.24 \mathrm{bcd}$ & $23.8 \pm 1.16 \mathrm{e}$ & $0.44 \pm 0.05 \mathrm{e}$ \\
\hline & Cd 1.5 & $2.6 \pm 0.24 a$ & $5.48 \pm 0.09 \mathrm{~cd}$ & $17.8 \pm 1.20 b c$ & $31.2 \pm 1.07 \mathrm{~d}$ & $0.70 \pm 0.07 \mathrm{bcd}$ \\
\hline & $\mathrm{Cd} 2.0$ & $2.8 \pm 0.20 a$ & $5.88 \pm 0.09 b$ & $19.8 \pm 0.66 \mathrm{ab}$ & $33.2 \pm 1.39 \mathrm{~cd}$ & $0.52 \pm 0.04 \mathrm{de}$ \\
\hline \multirow[t]{11}{*}{ D28 } & $\mathrm{Cd} 2.5$ & $2.8 \pm 0.37 a$ & $5.58 \pm 0.07 \mathrm{bcd}$ & $21.8 \pm 1.07 \mathrm{a}$ & $45.4 \pm 1.81 \mathrm{a}$ & $0.68 \pm 0.06 \mathrm{~cd}$ \\
\hline & Cd 3.0 & $2.8 \pm 0.20 a$ & $4.28 \pm 0.13 \mathrm{e}$ & $16.4 \pm 0.93 \mathrm{~cd}$ & $31.0 \pm 1.30 \mathrm{~d}$ & $0.44 \pm 0.09 \mathrm{e}$ \\
\hline & Cd 3.5 & $2.6 \pm 0.24 a$ & $4.18 \pm 0.09 \mathrm{e}$ & $17.6 \pm 1.08 b c$ & $32.8 \pm 1.24 \mathrm{~cd}$ & $0.74 \pm 0.05 b c$ \\
\hline & $\mathrm{Cd} 4.0$ & $2.8 \pm 0.37 a$ & $4.28 \pm 0.08 \mathrm{e}$ & $13.8 \pm 0.97 \mathrm{~d}$ & $23.8 \pm 1.07 \mathrm{e}$ & $0.90 \pm 0.05 b$ \\
\hline & $\mathrm{Cd} 4.5$ & $3.0 \pm 0.32 \mathrm{a}$ & $3.92 \pm 0.11$ ef & $22.0 \pm 1.41 \mathrm{a}$ & $30.2 \pm 1.28 d$ & $2.00 \pm 0.08 a$ \\
\hline & Cd 5.0 & $2.8 \pm 0.49 a$ & $3.72 \pm 0.13 f$ & $21.0 \pm 1.38 \mathrm{a}$ & $35.8 \pm 0.86 b c$ & $0.62 \pm 0.09 \mathrm{cde}$ \\
\hline & Cd 0.0 & $2.8 \pm 0.20 a$ & $6.38 \pm 0.04 b$ & $23.0 \pm 1.00 \mathrm{a}$ & $49.4 \pm 1.12 \mathrm{a}$ & $1.88 \pm 0.13 \mathrm{ab}$ \\
\hline & Cd 0.5 & $3.0 \pm 0.32 \mathrm{a}$ & $6.86 \pm 0.07 a$ & $22.2 \pm 0.80 a b$ & $39.4 \pm 1.08 c$ & $0.68 \pm 0.04$ ef \\
\hline & Cd 1.0 & $2.2 \pm 0.37 a$ & $5.70 \pm 0.15 \mathrm{~cd}$ & $16.2 \pm 1.66 \mathrm{~cd}$ & $24.2 \pm 1.07 \mathrm{e}$ & $0.48 \pm 0.04 \mathrm{f}$ \\
\hline & Cd 1.5 & $2.6 \pm 0.24 a$ & $5.80 \pm 0.13 c$ & $17.8 \pm 0.66 \mathrm{~cd}$ & $32.8 \pm 1.07 d$ & $0.70 \pm 0.07$ ef \\
\hline & Cd 2.0 & $2.8 \pm 0.37 a$ & $6.46 \pm 0.09 b$ & $20.0 \pm 1.00 a b c$ & $33.4 \pm 1.29 \mathrm{~d}$ & $0.58 \pm 0.05 f$ \\
\hline \multirow[t]{6}{*}{ D35 } & $\mathrm{Cd} 2.5$ & $2.8 \pm 0.49 a$ & $5.84 \pm 0.11 c$ & $22.0 \pm 1.00 a b$ & $45.4 \pm 1.63 b$ & $0.90 \pm 0.07 \mathrm{e}$ \\
\hline & Cd 3.0 & $2.8 \pm 0.37 a$ & $4.96 \pm 0.14 \mathrm{f}$ & $17.4 \pm 0.93 \mathrm{~cd}$ & $31.0 \pm 1.30 \mathrm{~d}$ & $1.70 \pm 0.14 b c$ \\
\hline & Cd 3.5 & $2.6 \pm 0.24 a$ & $5.20 \pm 0.14$ ef & $17.8 \pm 1.07 \mathrm{~cd}$ & $32.8 \pm 0.97 d$ & $2.04 \pm 0.08 a$ \\
\hline & Cd 4.0 & $2.8 \pm 0.37 a$ & $5.38 \pm 0.09 \mathrm{de}$ & $18.4 \pm 1.08 \mathrm{~cd}$ & $24.8 \pm 0.86 \mathrm{e}$ & $1.30 \pm 0.07 d$ \\
\hline & Cd 4.5 & $3.0 \pm 0.32 a$ & $4.14 \pm 0.13 \mathrm{~g}$ & $19.0 \pm 1.00 \mathrm{bcd}$ & $30.8 \pm 0.73 d$ & $1.62 \pm 0.06 c$ \\
\hline & Cd 5.0 & $2.8 \pm 0.37 \mathrm{a}$ & $4.24 \pm 0.09 \mathrm{~g}$ & $20.4 \pm 0.93 \mathrm{abc}$ & $29.8 \pm 1.71 \mathrm{~d}$ & $1.54 \pm 0.08 c$ \\
\hline \multicolumn{7}{|c|}{ F statistics } \\
\hline \multicolumn{2}{|c|}{ Source } & & & & & \\
\hline Days & 4,160 & $<1 \mathrm{~ns}$ & $193.51^{* *}$ & $24.94^{* *}$ & $58.47^{* *}$ & $9.41^{* *}$ \\
\hline Treat & 10,160 & $1.66 \mathrm{~ns}$ & $10.35^{\star \star}$ & $4.80^{\star \star}$ & $11.29^{\star *}$ & $2.30^{*}$ \\
\hline $\mathrm{D} \times \mathrm{T}$ & 40,160 & $<1 \mathrm{~ns}$ & $<1 \mathrm{~ns}$ & $<1 \mathrm{~ns}$ & $<1 \mathrm{~ns}$ & $1.61^{*}$ \\
\hline
\end{tabular}

Tabla 1. Número de ápices de brotes, hojas, taíces regeneradas y longitud del tallo y la raíz de Talinum portulacifolium expuestos a diferentes concentraciones de cadmio (Cd) a lo largo de un periodo de 35 días. *, **: Significativo a $\mathrm{p}<0,05$ y $\mathrm{p}<0,01$, respectivamente; ns: no significativo. Las medias de una columna seguidas de la misma(s) letra(s) no son diferentes significativamente (p>0,05) según la prueba de rango múltiple de Duncan.

Table 1. Number of shoot apices, leaves, roots regenerated and length of shoot and root of Talinum portulacifolium exposed to different concentrations of cadmium (Cd) over period of 35 days. *, **: Significant at $\mathrm{p}<0.05$ and $\mathrm{p}<0.01$ respectively; ns, not significant. Means in a column for a day followed by a same letter(s) are not significantly ( $>0.05)$ different according to Duncan's Multiple Range Test. 


\begin{tabular}{lccccc}
\hline Days (D) $\begin{array}{c}\text { Shoot apex } \\
\text { (per cutting) }\end{array}$ & $\begin{array}{c}\text { Shoot length } \\
\text { (cm) }\end{array}$ & $\begin{array}{c}\text { Leaf number } \\
\text { (per cutting) }\end{array}$ & $\begin{array}{c}\text { Root number } \\
\text { (per cutting) }\end{array}$ & $\begin{array}{c}\text { Total root } \\
\text { length }(\mathrm{cm})\end{array}$ \\
\hline D7 & $0.075 \mathrm{~ns}$ & $-0.820^{* *}$ & $-0.019 \mathrm{~ns}$ & $-0.772^{* *}$ & $-0.656^{*}$ \\
D14 & $-0.050 \mathrm{~ns}$ & $-0.908^{* * *}$ & $-0.694^{*}$ & $-0.818^{* *}$ & $-0.085 \mathrm{~ns}$ \\
D21 & $-0.246 \mathrm{~ns}$ & $-0.244 \mathrm{~ns}$ & $-0.224 \mathrm{~ns}$ & $-0.460 \mathrm{~ns}$ & $-0.303 \mathrm{~ns}$ \\
D28 & $-0.313 \mathrm{~ns}$ & $-0.889^{* * *}$ & $-0.210 \mathrm{~ns}$ & $-0.381 \mathrm{~ns}$ & $-0.232 \mathrm{~ns}$ \\
D35 & $0.175 \mathrm{~ns}$ & $-0.873^{* * *}$ & $-0.296 \mathrm{~ns}$ & $-0.510 \mathrm{~ns}$ & $0.441 \mathrm{~ns}$ \\
\hline
\end{tabular}

Tabla 2. Correlación de Pearson para la concentración de cadmio y el número de ápices de brotes, longitud del brote, hoja, número y longitud total de raíces durante diferentes puntos temporales $(\mathrm{n}=10) .{ }^{*}, * *, * * *$ : Significativo a $\mathrm{p}<0,05, \mathrm{p}<0,01, \mathrm{p}<0,001$; ns: no significativo.

Table 2. Pearson's correlation for the cadmium concentration and the number of shoot apices, shoot length, leaf and numbers and total root length during different time points $(\mathrm{n}=10) .{ }^{*}, * *, * *$ : Significant at $\mathrm{p}<0.05, \mathrm{p}<0.01, \mathrm{p}<0.001$ respectively. $\mathrm{ns}$, not significant.

\begin{tabular}{|c|c|c|c|c|c|c|}
\hline \multirow{2}{*}{$\begin{array}{c}\mathrm{Cd} \\
(\mathrm{ppm})\end{array}$} & \multicolumn{3}{|c|}{ Fresh weight (mg) } & \multicolumn{3}{|c|}{ Dry weight (mg) } \\
\hline & Leaf & Stem & Root & Leaf & Stem & Root \\
\hline $\mathrm{Cd} 0.0$ & $1582.31 \pm 119.22 \mathrm{a}$ & $5351.62 \pm 52.10 \mathrm{a}$ & $421.35 \pm 72.09 a$ & $1012.83 \pm 142.76 a$ & $3302.43 \pm 316.81 a$ & $102.43 \pm 17.12$ \\
\hline d 0.5 & $1363.71 \pm 52.91 \mathrm{ab}$ & $5802.74 \pm 75.64 a$ & $62 \pm 4$ & $4 \pm 10$ & $3005.48 \pm$ & $35 \pm$ \\
\hline d 1.0 & $881.51 \pm 114.6$ & $3681.77 \pm 32.62 b$ & $278.93 \pm 58$ & $3 \pm 4$ & 2378. & $41 \pm$ \\
\hline d 1.5 & $1024.41 \pm 180.59 \mathrm{bc}$ & $\pm 271.27 b$ & $40 \pm 7$ & $8 \pm 83.1$ & 221 & $6 a$ \\
\hline d 2.0 & 1011. & $\pm 488.70 \mathrm{~b}$ & $28 \pm 1$ & $3 \pm 77$ & 2501.6 & 8 a \\
\hline $\mathrm{Cd} 2.5$ & $1213.21 \pm 1$ & & & & & $8 a$ \\
\hline Cd 3.0 & $564.16 \pm 10.7$ & 3563 & 8 a & $12 \pm 20$ & 213 & $44 \mathrm{a}$ \\
\hline Cd 3.5 & 63 & $\pm 529.39 b$ & a & & & $3 a$ \\
\hline $\mathrm{Cd} 4.0$ & $850.14 \pm 98.41$ & $3807.25 \pm 418.35 b$ & $204.17 \pm 63$ & $18 \pm 1$ & 2303. & 66 a \\
\hline $\mathrm{Cd} 4.5$ & $632.88 \pm 9.73 d$ & $3958.83 \pm 162.38 b$ & $236.15 \pm 64.70 \mathrm{a}$ & $2.54 \pm 2.6$ & $\pm 27.59 \mathrm{abc}$ & $101.24 \pm 8$ \\
\hline Cd 5.0 & $854.32 \pm 68.19 \mathrm{~cd}$ & $4370.18 \pm 460.65 b$ & $203.84 \pm 65.75 \mathrm{a}$ & $713.65 \pm 72.56 c$ & $3140.94 \pm 30.23 a$ & $101.27 \pm 8.70 \mathrm{a}$ \\
\hline & $9.516^{* \star *}$ & $5.336^{* * *}$ & $1.870 \mathrm{~ns}$ & $5.768^{* * *}$ & $5.420^{* * *}$ & $0.003 \mathrm{~ns}$ \\
\hline$=11$ & $-0.744^{* *}$ & $-0.529 n s$ & $-0.507 n s$ & $-0.578 n s$ & $-0.102 n s$ & $-0.490 \mathrm{~ns}$ \\
\hline
\end{tabular}

Tabla 3. Pesos freso y seco de Talinum portulacifolium de partes de plantas expuestos a diferentes concentraciones de cadmio (Cd) y su relacion. *, **: Significativo a $\mathrm{p}<0,05$ y $\mathrm{p}<0,01$, respectivamente; ns: no significativo. Las medias de una columna seguidas de la misma(s) letra(s) no son diferentes significativamente $(\mathrm{p}>0,05)$ según la prueba de rango múltiple de Duncan.

Table 3. Fresh and dry weights of Talinum portulacifolium plant parts exposed to different concentrations of cadmium (Cd) and their relationship. *, **: Significant at $\mathrm{p}<0.05$ and $\mathrm{p}<0.01$ respectively; ns, not significant. Means in a column for a day followed by a same letter(s) are not significantly ( $>0.05$ ) different according to Duncan's Multiple Range Test.

leaves and roots were observed in $\mathrm{Cd} 0.0$ compared to other concentrations of $\mathrm{Cd}$. Total root length in Cd4.5 decreased by $49 \%$ compared to Cd0.0 (Table 1). Shoot length, leaf and root numbers were significantly and negatively correlated to the concentration of the $\mathrm{Cd}$ in the nutrient solution (Table 2).

At D21, maximum shoot length was in $\mathrm{Cd} 0.5$ and more number of leaves was observed in Cd0.0. Root numbers of Cd1.0 showed a $57 \%$ decrease over Cd0.0 (Table 1). Total root length ranged between $1.62 \mathrm{~cm}(\mathrm{Cd} 0.0)$ and $0.40 \mathrm{~cm}$ (Cd3.0) among the different concentrations of $\mathrm{Cd}$. No significant correlation existed between the concentrations of $\mathrm{Cd}$ and different growth parameters observed on D21 (Table 2).

Cuttings in $\mathrm{Cd} 0.5$ had the maximum shoot length and minimum shoot length was observed in Cd5.0 on D28. There was no significant variation in the leaf number among the various treatments.

The number of roots ranged from $48.4(\mathrm{Cd} 0.0)$ to $23.8(\mathrm{Cd} 1.0$ and $\mathrm{Cd} 4.0)$ and the root numbers in Cd1.0 and Cd4.0 were 51\% lower than Cd0.0. Total root length was maximum in $\mathrm{Cd} 0.0$ and minimum in $\mathrm{Cd} 1.0$ and $\mathrm{Cd} 3.0$. Increasing concen- trations of Cd significantly and inversely influenced the shoot length (Table 2).

On D35 shoot numbers were higher in $\mathrm{Cd} 0.5$ compared to $\mathrm{Cd} 0.0$, and it gradually decreased with increasing concentrations of $\mathrm{Cd}$. The maximum number of leaves and roots was observed in Cd0.0, and minimum occurred in Cd1.0. The root length ranged between $2.04 \mathrm{~cm}(\mathrm{Cd} 3.5)$ and 0.48 $\mathrm{cm}$ (Cd1.0) (Table 1). A significant negative correlation existed between the shoot length and increasing concentrations of Cd (Table 2).

\section{Plant fresh and dry weights}

Different concentrations of Cd significantly influenced the fresh and dry weights of stem and leaf but not root (Table 3). Changes in the fresh and dry weights of plant parts were not linear except for the leaf fresh weight which was significantly and negatively correlated to $\mathrm{Cd}$ concentrations. Leaf fresh weight was maximum in $\mathrm{Cd} 0.0$ and minimum in $\mathrm{Cd} 3.0 \mathrm{ppm}(0.56 \mathrm{~g})$. The decline in leaf fresh weight in $\mathrm{Cd}$ spiked solutions ranged from $14-65 \%$. Maximum fresh weight of stems was recorded in $\mathrm{Cd} 0.5$ cuttings which were $8 \%$ higher than Cd0.0 (Table 3). While the fresh 
weight of stems in other Cd spiked solutions was $18-36 \%$ lower than that of Cd0.0. Though the 10$62 \%$ decline in root fresh weighs among Cd treatments were not significant, a $5 \%$ and $10 \%$ in root fresh weight was evident in cuttings raised in $\mathrm{Cd} 2.0$ and $\mathrm{Cd} 2.5$ respectively. Not much variation was observed in root fresh weight among different treatments. The maximum dry weight of leaves and shoots occurred in $\mathrm{Cd} 0.0$ compared to different concentrations of $\mathrm{Cd}$. The dry weights of leaf, stem and roots in different concentrations of $\mathrm{Cd}$ spiked solutions were $5-35 \%, 23-56 \%$ and 0.20 $1.56 \%$ lower than Cd0.0 (Table 3 ).

\section{Morphological changes}

At higher concentrations of $\mathrm{Cd}(\mathrm{Cd} 5.0)$ leaves weathered off and chlorosis of matured leaves was evident. Cadmium also caused stem decay, browning of roots and stunted root and shoot growth in $T$. portulacifolium, and necrotic spots on the leaves. Pink colouration of the metal solution was observed after four days, in which the stem cuttings of $T$. portulacifolium were immersed. The intensity of colouring increased over a period of the next seven days and gradually disappeared during the later stages of plant growth.

\section{Accumulation of $\mathrm{Cd}$ in plant parts}

Significant variation $(p<0.001)$ existed between the accumulation of $\mathrm{Cd}$ in the stems $\left(\mathrm{F}_{10,22}=\right.$ 28.718) and leaf $\left(\mathrm{F}_{10,22}=12.810\right)$ of $T$. portulacifolium at various concentrations of $\mathrm{Cd}$ (Fig. 2). Accumulation of $\mathrm{Cd}$ in the T. portulacifolium stem increased linearly with the increasing concentrations of $\mathrm{Cd}$ in the nutrient solution, with the highest accumulation at $\mathrm{Cd} 4.0 \mathrm{ppm}$. In contrast changes in $\mathrm{Cd}$ accumulation in the $T$. portulacifolium leaves were not linear with concentrations of $\mathrm{Cd}$ in the nutrient solution and maximum accumulation occurred at $\mathrm{Cd} 3.0$ (Fig. 2). Cadmium concentrations in leaves was not correlated to $\mathrm{Cd}$ concentration in stems $(\mathrm{r}=-0.071 ; \mathrm{p}>0.05$; $\mathrm{n}=10$ ).

\section{Tolerance index}

The Ti of T. portulacifolium ranged from $59.09 \%$ (Cd3.0) to $87.72 \%$ (Cd0.5) (Fig. 3) and was not related to the concentrations of $\mathrm{Cd}$ in the nutrient solution $(\mathrm{r}=0.118 ; \mathrm{p}>0.05 ; \mathrm{n}=10)$. The Ti of plants in $\mathrm{Cd} 0.5$ was $0.51 \%-32.64 \%$ higher than plants in other $\mathrm{Cd}$ treatments. The Ti was significantly and negatively correlated to $\mathrm{Cd}$ content in leaves

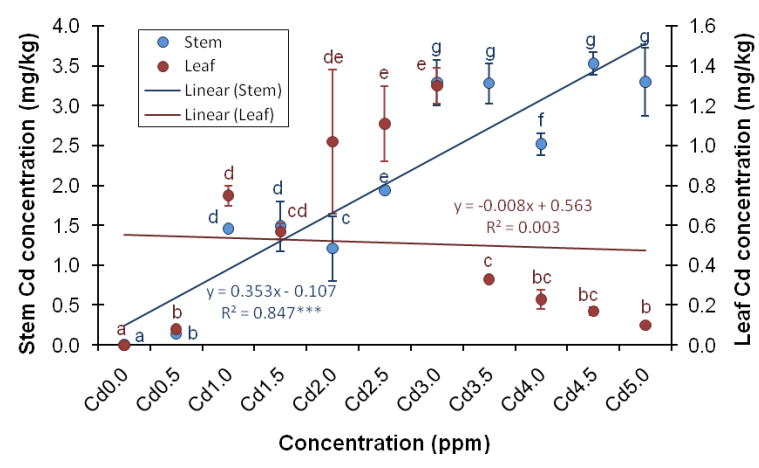

Figura 2. Contenido de cadmio en hojas y tallos de Talinum portulacifolium esquejes expuestos a diferentes concentraciones de Cd en cultivo hidropónico y la relación entre ambos. Puntos \pm error estándar con la misma(s) letra(s) no son diferentes significativamente $(\mathrm{p}>0,05)$ según la prueba de rango múltiple de Duncan. *** Significatico a $\mathrm{p}<0,001$.

Figure 2. Cadmium $(\mathrm{Cd})$ content in leaves and stems of Talinum portulacifolium stem cuttings exposed to different concentrations of $\mathrm{Cd}$ in hydroponic culture and their relationship. Points \pm standard error for a variable bearing same letter(s) are not significantly different ( $p>0.05$ ) according to Duncan's Multiple Range Test. ***Significant at $\mathrm{p}<0.001$.

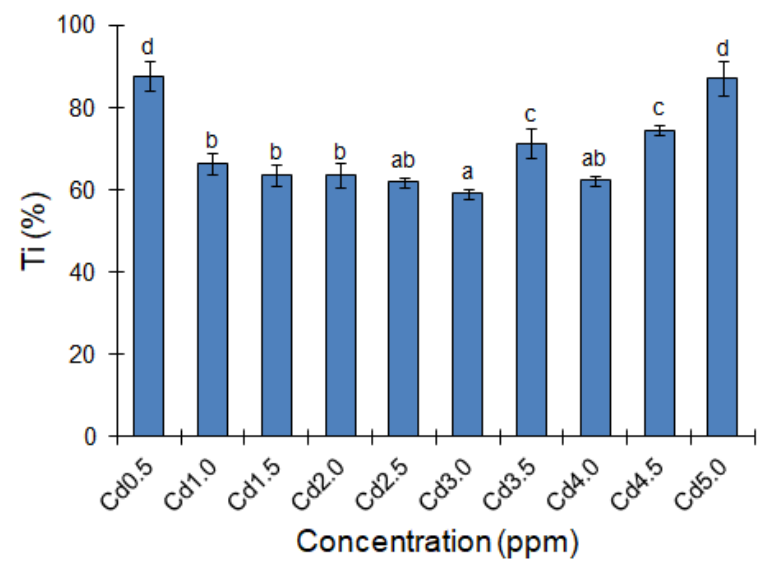

Figura 3. Índice de tolerancia (Ti) de esquejes de Talinum portulacifolium creciendo a distintas de cadmio (Cd) en cultivo hidropónico. Las barras de error indican error estándar. Barras con la misma(s) letra(s) no son significativamente diferentes $(\mathrm{p}>0,05)$ según la prueba de rango múltiple de Duncan.

Figure 3. Tolerance index (Ti) of Talinum portulacifolium stem cuttings grown in different concentrations of cadmium $(\mathrm{Cd})$ in hydroponic culture. Error bars indicate \pm standard error. Bars bearing same letter(s) are not significantly different $(p>0.05)$ according to Duncan's Multiple Range Test.

$(\mathrm{r}=-0.758 ; \mathrm{p}<0.01 ; \mathrm{n}=10)$ and not in stems $(\mathrm{r}=-$ $0.081 ; \mathrm{p}>0.05 ; \mathrm{n}=10)$.

\section{Discussion}

\section{Effect on the regeneration of stem cuttings}

The results of the present study show that different concentrations of $\mathrm{Cd}$ affect the regeneration of the stem cuttings of T. portulacifolium. Once sep- 
arated from the mother plant, hormones such as auxins, jasmonates, and ethylene start accumulating at the cut region and eventually play varied roles in the development of the adventitious roots and regeneration of the cuttings (Steffens \& Rasmussen 2016). The cuttings first physiological response to the detachment from the mother plant is first by the formation of the callus and then by the roots. However, these two are independent activities (Dole \& Gibson 2006). Though rootless stem cuttings of $T$. portulacifolium developed callus within three days in all the $\mathrm{Cd}$ concentrations, this physiological process was not observed in previous studies examining the influence of $\mathrm{Cd}$ on the stem cuttings of Talinum (Rajkumar et al. 2009) or Portulaca L. species (Mohanapriya et al. 2006, Thangavel \& Subburam 1998). Nevertheless, callus formation in response to exposure to different concentrations of zinc $(\mathrm{Zn})$ has been recently reported in T. portulacifolium (Muthukumar et al. 2018). However, later changes in hormone signalling with the increased presence of cytokinin and strigolactone prevent callus proliferation and initiate adventitious root development (Steffens \& Rasmussen 2016).

The initiation of roots within seven days in the nutrient solutions that were unspiked or spiked with low concentrations of $\mathrm{Cd}$ (up to Cd1.5) is in accordance with the observations of Kumar \& Prasad (2010) where T. portulacifolium stem cuttings grown in hydroponic medium developed roots within a week. Nevertheless, the delay in root initiation with increasing concentrations of $\mathrm{Cd}$ in the solution is in line with the observations of Rajkumar et al. (2009) who also observed a delay in the initiation of roots in T. fruticosum (as Talinum triangulare (Jacquin) Willdenow) with increasing concentrations of $\mathrm{Cd}(0.5-3.0 \mathrm{mg} / \mathrm{L})$. Moreover, in contrast to the observations of Rajkumar et al. (2009), where stem cuttings of $T$. fruticosum failed to initiate roots beyond $3.0 \mathrm{mg} / \mathrm{L}$ of $\mathrm{Cd}$, root initiation in $T$. portulacifolium stem cuttings occurred even in the concentration of 5.0 $\mathrm{mg} / \mathrm{L}$ tested. Increasing concentrations of other heavy metals like copper $(\mathrm{Cu})$ and $\mathrm{Zn}$ also delays root initiation in Portulaca oleracea L. and T. portulacifolium respectively (Mohanapriya et al. 2006, Muthukumar et al. 2018). Degradation of auxin may be liable for the Cd-induced differences in the rooting ability of $T$. portulacifolium stem cuttings. For instance, exposure of poplars (Populus $\times$ canescens (Aiton) Sm.) stem cuttings to $\mathrm{Cd}$ upregulated the GH3 enzyme activities responsible for the removal of auxins from the dynamic pool through conjugation which in turn reduced the concentration of auxins in the stem (Elobeid et al. 2011). The delay in root initiation can also be due to the reduction in the synthesis of cytokinin, the hormone responsible for the initiation of adventitious roots (Vysotskaya et al. 2007), thereby affecting the supply of nutrients consequent to the inhibitory effect of metals on the metabolic pathways (Hashem 2014).

Compared to roots leaves sprouted without much delay in all concentrations of $\mathrm{Cd}$ and emerged on the stem cuttings on the $2^{\text {nd }}$ or $3^{\text {rd }}$ day. A similar response has also been noted in $P$. oleracea where leaves sprouted on the $3^{\text {rd }}$ day on the stems cuttings exposed to different sources and concentrations of $\mathrm{Cu}$ (Mohanapriya et al. 2006). In contrast, sprouting happened on the $4^{\text {th }}$ day after exposure to $\mathrm{Cd}$ in $T$. fruticosum stem cuttings (Rajkumar et al. 2009). In spite of high mobility of $\mathrm{Cd}$ in plants, sprouting occurred even in the highest concentration of Cd tested (Eze et al. 2014). This implies the lack of buildup of critical concentrations of $\mathrm{Cd}$ in the stems are adequate enough to affect the regeneration of the cutting during the early stages of exposure. Moreover, as roots are in direct contact with the metal source they exhibit early symptoms to metal exposure than the shoots (Mohanapriya et al. 2006). Unrooted stem cuttings take up a minimal amount of water and nutrients directly through the cut surface (Alem 2010, Santos et al. 2009). This could also be true for the uptake of HMs like Cd by unrooted stem cuttings resulting in weak response to metal exposure during the initial stages.

The reduction in the number of shoot buds and leaves with increasing concentrations of $\mathrm{Cd}$ in $T$. portulacifolium stem cuttings may be due to the inhibition of cytokinin synthesis by $\mathrm{Cd}$ (Hashem 2014) since the role of this phytohormone on cell division and shoot morphogenesis are well established (Kieber \& Schaller 2018). Similar observations were also reported for $P$. oleracea and $T$. portulacifolium for other $\mathrm{HMs}$ like $\mathrm{Cu}$ and $\mathrm{Zn}$ (Mohanapriya et al. 2006, Muthukumar et al. 2018). The reduction of leaf number and area was also evident in response to $\mathrm{Cd}$ toxicity in hydroponic experiments done by Zacchini et al. (2009) with poplar and willow clones. The reduction in shoot and root lengths of $T$. portulacifolium stem cuttings in response to $\mathrm{Cd}$ exposure is similar to 
the observations of Lai et al. (2016) where different concentrations of $\mathrm{Cd}$ reduced the growth of Impatiens walleriana Hook.f. rooted stem cuttings exposed to different concentrations of $\mathrm{Cd}$. The reduced plant elongation may be due to the direct toxic effect of $\mathrm{Cd}$ on the membrane integrity and cell division. Moreover, a reduction in the root respiration and protein synthesis in addition to plant photosynthesis might have also contributed to the slow growth of $T$. portulacifolium roots and shoots in response to Cd toxicity (Agarwal et al. 1987). The slow development of roots indicates metal penetration and accumulation in roots (Mohanapriya et al. 2006). Browning of roots and stunned root and shoot growth was also reported by Koleva et al. (2010) where Cd inhibited root length and induced browning in durum wheat.

\section{Effect of Cd on plant fresh and dry weights}

Exposure to $\mathrm{Cd}$ affected the fresh and dry weights of stem cuttings corroborating the observations in I. walleriana and Salix triandroides W.P. Fang stem cuttings exposed to Cd (Lai et al. 2017, Yao et al. 2018). Plant biomass can reduce at an organ level or whole plant level when the toxic elements are absorbed by plants (Emamverdian et al. 2015). This decline in response to the uptake of toxic elements could be due to the inhibition of the metabolic process that is related to plant growth. For instance, recent studies have shown that $\mathrm{Cd}$ can alter the auxin (IAA) homeostasis in the root system through its detrimental effects on the IAA biosynthetic genes as well as the distribution of the hormone can affect root growth (Bruno et al. 2017, Ronzan et al. 2018).

\section{Tolerance to $\mathbf{C d}$}

Tolerance often circumscribed as the extent to which a plant can resist exposure to increasing concentrations of HMs without displaying any phytotoxicity (Zha et al. 2004). One way of assessing tolerance to HMs is through observing changes in plant growth with increasing contaminant exposure i.e., Ti. Though T. portulacifolium exhibited a certain degree of tolerance for all the fitness parameters measured within the tested $\mathrm{Cd}$ concentrations, a decline in plant growth was evident with increasing levels of $\mathrm{Cd}$. The negative relation between $\mathrm{Ti}$ and concentration of $\mathrm{Cd}$ in $T$. portulacifolium leaves is in accordance with Henson et al. (2013) who also reported a negative correlation between $\mathrm{Ti}$ and shoot $\mathrm{Cd}$ concentrations in Chamaecrista fasciculata (Michx.) Greene partridge pea. Normally metal hyperaccumulators are characterized by improved tolerance accomplished through internal detoxification (Pollard et al. 2002). Nevertheless, T. portulacifolium could not be designated as a hyperaccumulator as the plants accumulated less than $100 \mathrm{mg} / \mathrm{kg}$ of Cd under the present set of growth conditions (Henson et al. 2013). The phytotoxic effects of $\mathrm{Cd}$ on $T$. portulacifolium were well pronounced as the plants showed various morphological changes like wilting and withering of leaves in addition to the necrotic spots. The accelerated senescence caused by $\mathrm{Cd}$ in leaves may be attributed to the increased cell membrane permeability (Langille \& MacLean 1976).

\section{Morphological changes}

Exposure to high concentrations of Cd-induced chlorosis in leaves as $\mathrm{Cd}$ can affect the biosynthesis of chloroplasts as well as the chloroplast structure resulting in a decreased number of chloroplasts per cell and reduced photosynthesis (Sun et al. 2015). This is in accordance with Koleva et al. (2010) where Cd-induced chlorosis in the leaves of Triticum durum L. has been reported. Moreover, excess concentrations of $\mathrm{Cd}$ are known to induce changes in cell size and interfere with the cell division (Baryla et al. 2001). The toxicity symptoms of $\mathrm{Cd}$ are indicated by the appearance of necrotic spots on the leaves, poor branching of roots and root browning (Das et al. 1997).

The pink colouration of the metal solution during early stages of cutting regeneration indicates the exudation of plant pigments as noted by Rajkumar et al. (2009). Earlier studies suggest that Talinum accumulate greater concentrations of anthocyanins in response to heavy metal exposure (Kumar et al. 2012). Anthocyanins are dominant secondary metabolites that protect plants against oxidative stresses by quenching the free radical ions through the donation of phenolic hydrogen atoms (Hernández et al. 2009). Furthermore, anthocyanins can also react with metals forming complexes thereby protecting plants from various abiotic stresses (Castaneda-Ovando et al. 2009).

\section{Cd accumulation and its effect on consumption and therapeutic value}

According to FAO/WHO (2014), the permissible limit for $\mathrm{Cd}$ is $0.05 \mathrm{mg} / \mathrm{kg}$ for vegetables and $0.2 \mathrm{mg} / \mathrm{kg}$ for leafy vegetables. Based on the ob- 
servations of the present study $T$. portulacifolium accumulated up to 18 folds higher $\mathrm{Cd}$ in the stems and six folds higher $\mathrm{Cd}$ in the leaves than the permissible limits for leafy vegetables. This is accordance with studies where species of Talinum growing in soils contaminated with $\mathrm{Cd}$ like the dumpsites accumulated enormous levels of $\mathrm{Cd}$ (2$72 \mathrm{mg} / \mathrm{kg}$ ) in their shoots (Obasi et al. 2009, 2013, 2015). The concentration of $\mathrm{Cd}$ in $T$. portulacifolium is also several times higher compared to the concentration of $\mathrm{Cd}$ reported for other leafy vegetables from different parts of the globe (Huang et al. 2017). As the major source of $\mathrm{Cd}$ exposure in humans occurs through the consumption of leafy vegetable either in cooked or raw forms and leafy vegetables acquire and accumulate more $\mathrm{Cd}$ than other crops. Chronic exposure to low concentrations of $\mathrm{Cd}$ from non-dietary sources can also result in organ toxicity especially nephrotoxicity in mammals (Thévenod \& Lee 2013). Moreover, some recent studies have shown that the raw materials used in the preparation of herbal drugs are the major source of HMs in plant-based pharmaceutical products (Dghaim et al. 2015, Nessa et al. 2016). Hence it is important to grow this leafy vegetable in uncontaminated soils or collect the plant materials from unpolluted areas for therapeutic preparations (Girisha \& Ragavendra 2009, Huang et al. 2017).

\section{Conclusions}

Pollution of soil and water by $\mathrm{Cd}$ is a global problem that affects both plant and human health mainly in Africa and Asia. Our results show that $\mathrm{Cd}$ interferes with the regeneration potential of $T$. portulacifolium stem cuttings by affecting the root initiation and plant development. From the results, it is also clear that $T$. portulacifolium can accumulate great concentrations of $\mathrm{Cd}$ if available in the growing medium. Despite the accumulation of appreciable quantity of $\mathrm{Cd}$ than those prescribed for leafy vegetables, $T$. portulacifolium is not a hyperaccumulator of $\mathrm{Cd}$ under the studied conditions. Therefore caution is necessary while consuming shoots of $T$. portulacifolium originating from unknown sources. The same is also true for using $T$. portulacifolium as a therapeutic agent.

\section{References}

Adefemi OS, Ibigbami OA \& Awokunmi EE. 2012. Level of heavy metals in some edible plants collected from selected dumpsites in Ekiti State, Nigeria. Global Advanced Research Journal of Environmental Science and Toxicology1(5): 13-26.

Agarwal K, Sharma A \& Talukder G. 1987. Copper toxicity in plant cellular systems. Nucleus 30(3): 131158.

Alem P. 2010. Unrooted stem cutting physiology; water use and leaf gas exchange of severed stem cuttings. USA: Clemson University. Masters Thesis.

Antosiewicz DM. 1993. Mineral status of dicotyledonous crop plants in relation to their constitutional tolerance to lead. Environmental and Experimental Botany 33(4): 575-589. https://doi.org/10.1016/0098-8472 (93) $90032-B$

Babayemi JO, Olafimihan OH \& Nwude DO. 2017. Assessment of heavy metals in water leaf from various sources in Ota, Nigeria. Journal of Applied Sciences and Environmental Management 21(6): 1163-1168. https://dx.doi.org/10.4314/jasem.v21i6.29

Baryla A, Carrier P, Franck F, Coulomb C, Sahut C \& Havaux M. 2001. Leaf chlorosis in oilseed rape plants (Brassica napus) grown on cadmium-polluted soil: causes and consequences for photosynthesis and growth. Planta 212(5-6): 696-709. https://doi. org/10.1007/s004250000439

Bruno L, Pacenza M, Forgione I, Lamerton LR, Greco M, Chiappetta A \& Bitonti MB. 2017. In Arabidopsis thaliana cadmium impact on the growth of primary root by altering SCR expression and auxin-cytokinin cross-talk. Frontiers in Plant Science 8: 1323. https://doi.org/10.3389/fpls.2017.01323

Castaneda-Ovando A, de Lourdes Pacheco-Hernández M, Páez-Hernández ME, Rodríguez JA \& GalánVidal CA. 2009. Chemical studies of anthocyanins: A review. Food chemistry 113(4): 859-871. https://doi. org/10.1016/j.foodchem.2008.09.001

Clemens S, Aarts MG, Thomine S, Verbruggen N. 2013. Plant science: the key to preventing slow cadmium poisoning. Trends Plant Science 18(2): 92-99. https://doi.org/10.1016/j.tplants.2012.08.003

Das P, Samantaray S \& Rout G. 1997. Studies on cadmium toxicity in plants: a review. Environmental Pollution 98(1): 29-36. https://doi.org/10.1016/S02697491(97)00110-3

de Souza GG, Pinheiro AL, Silva JA, Veroneze-Júnior V, Carvalho M, Bertoli AC, Barbosa S \& de Souza TC. 2018. Morpho-physiological tolerance mechanisms of Talinum patens to lead. Water Air and Soil Pollution 229:4. https://doi.org/10.1007/s11270-017-3658$\underline{0}$

Dghaim R, Al Khatib S, Rasool H \& Ali Khan M. 2015. Determination of heavy metals concentration in traditional herbs commonly consumed in the United Arab Emirates. Journal of Environmental and Public Health. 2015: 1-6. http://dx.doi.org/10.1155/2015/ 9 $\underline{73878}$

Dole JM \& Gibson JL. 2006. Cutting propagation: a guide to propagating and producing floriculture crops. Batavia, USA: Ball Publishing.

Dushenkov V, Kumar PN, Motto H \& Raskin I. 1995. Rhizofiltration: the use of plants to remove heavy metals from aqueous streams. Environmental Science and Technology 29(5): 1239-1245. https://doi. 


\section{org/10.1021/es00005a015}

Ebong GA, Etuk HS \& Dan EU. 2018. Distribution, pollution index and associated health risk of trace metals in waste-impacted soils within Akwa lbom State, Nigeria. Geosystem Engineering 21(3): 121-34. https:// doi.org/10.1080/12269328.2017.1376291

Elobeid M, Göbel C, Feussner I \& Polle A. 2011. Cadmium interferes with auxin physiology and lignification in poplar. Journal of Experimental Botany 63(3): 1413-1421. https://doi.org/10.1093/jxb/err384

Emamverdian A, Ding Y, Mokhberdoran F \& Xie Y. 2015. Heavy metal stress and some mechanisms of plant defense response. The Scientific World Journal 2015, Article ID 756120, 18 pages. https://doi.org/ $10.1155 / 2015 / 756120$

Eze MO \& Ekanem EO. 2014. Bioaccumulation and mobility of cadmium $(\mathrm{Cd})$, lead $(\mathrm{Pb})$ and zinc $(\mathrm{Zn})$ in green spinach grown on dumpsite soils of different $\mathrm{pH}$ levels. Bulletin of Environment, Pharmacology and Life Sciences 4(1): 85-91. https://doi.org/10. 1007/s10661-018-7051-2

FAO/WHO. 2014. General standards for contaminants and toxins in food and feed (CODEX STAN 1931995). Available in www.fao.org/input/download/ standards/17/CXS_193e_2015.pdf (Accessed 29 April 2019).

Friberg L. 2018. Cadmium in the Environment. Boca Raton: CRC Press.

Friberg LT, Elinder CG, Kjellstrom T \& Nordberg GF. 2019. Cadmium and health: A toxicological and epidemiological appraisal: Volume 2: Effects and response. USA: CRC Press.

Girisha ST \& Ragavendra VB. 2009. Accumulation of heavy metals in leafy vegetables grown in urban areas by using sewage water and its effect. Archives of Phytopathology and Plant Protection 42(10): 956959. https://doi.org/10.1080/03235400701543806

Hashem HA. 2014. Cadmium toxicity induces lipid peroxidation and alters cytokinin content and antioxidant enzyme activities in soybean. Botany 92(1): 1-7. https://doi.org/10.1139/cjb-2013-0164

He S, Yang X, He Z \& Baligar VC. 2017. Morphological and physiological responses of plants to cadmium toxicity: A review. Pedosphere 27(3): 421-438. https://doi.org/10.1016/S1002-0160(17)60339-4

Henson TM, Cory W \& Rutter MT. 2013. Extensive variation in cadmium tolerance and accumulation among populations of Chamaecrista fasciculata. PLoS ONE 8(5): e63200. https://doi.org/10.1371/ journal.pone. 0063200

Hernández I, Alegre L, Van Breusegem F \& MunnéBosch S. 2009. How relevant are flavonoids as antioxidants in plants? Trends in Plant Science 14(3): 125-132. https://doi.org/10.1016/j.tplants.2008.12.003

Huang Y, He C, Shen C, Guo J, Mubeen S, Yuan J \& Yang Z. 2017. Toxicity of cadmium and its health risks from leafy vegetable consumption. Food and Function 8: 1373-1401. https://doi.org/10.1016/j. tplants.2008.12.003

Jackson AP \& Alloway BJ. 2017. The transfer of cadmium from agricultural soils to the human food chain. In Biogeochemistry of trace metals (Adriano DC, ed.). Boca Raton: CRC Press, pp. 121-170.

Jansen PCM. 2004. Talinum portulacifolium (Forssk.) Asch. ex Schweinf. Record from PROTA4U (Grubben GJH \& Denton OA, Eds.). PROTA (Plant Resources of Tropical Africa / Ressources végétales de l'Afrique tropicale), Wageningen, Netherlands. Available in http://www.prota4u.org/search.asp (Accessed 29 April 2019).

Khan MA, Khan S, Khan A \& Alam M. 2017. Soil contamination with cadmium, consequences and remediation using organic amendments. Science of the Total Environment. 601-602: 1591-605. https://doi. org/10.1016/j.scitotenv.2017.06.030

Kieber JJ \& Schaller GE. 2018. Cytokinin signaling in plant development. Development 145. https://doi. org/10.1242/dev.149344

Koleva L, Semerdjieva I, Nikolova A \& Vassilev A. 2010. Comparative morphological and histological study on zinc- and cadmium-treated durum wheat plants with similar growth inhibition. General and Applied Plant Physiology 36(1-2): 8-11.

Kubier A, Wilkin RT \& Pichler T. 2019. Cadmium in soils and groundwater: A review. Applied Geochemistry 108:104388. https://doi.org/10.1016/j.apgeochem.2 $\underline{019.104388}$

Kumar A \& Prasad MN. 2015. Lead-induced toxicity and interference in chlorophyll fluorescence in Talinum triangulare grown hydroponically. Photosynthetica 53: 66-71. https://doi.org/10.1007/s11099-015-0091$\underline{8}$

Kumar A \& Prasad MNV. 2010. Propagation of Talinum cuneifolium L. (Portulacaceae), an ornamental plant and leafy vegetable, by stem cuttings. Floriculture and Ornamental Biotechnology 4(S1): 68-71.

Kumar A, Prasad MNV \& Sytar O. 2012. Lead toxicity, defense strategies and associated indicative biomarkers in Talinum triangulare grown hydroponically. Chemosphere 89(9): 1056-1065. https://doi.org/10. 1016/j.chemosphere.2012.05.070

Lai HY \& Cai MC. 2016. Effects of extended growth periods on subcellular distribution, chemical forms, and the translocation of cadmium in Impatiens walleriana. International Journal of Phytoremediation 18(3): 228-234. https://doi.org/10.1080/15226514.2015.10 $\underline{73677}$

Lai HY, Lam CM, Wang WZ \& Ji YJ. 2017. Cadmium uptake by cuttings of Impatiens walleriana in response to different cadmium concentrations and growth periods. Bulletin of Environmental Contamination and Toxicology 98(3): 317-322. https://doi.org/10.1007/ s00128-016-1874-8

Langille WM \& MacLean KS. 1976. Some essential nutrient elements in forest plants as related to species, plant part, season and location. Plant and Soil 45(1): 17-26. https://doi.org/10.1007/BF00011125

McBride MB, Martinez CE \& Kim B. 2016. Zn, Cd, S and trace metal bioaccumulation in willow (Salix spp.) cultivars grown hydroponically. International Journal of Phytoremediation 18(12): 1178-1186. https://doi. org/10.1080/15226514.2016.1189401

Mohanapriya S, Senthilkumar P, Sivakumar S, Dineshkumar M \& Subbhuraam V. 2006. Effects of copper 
sulfate and copper nitrate in aquatic medium on the restoration potential and accumulation of copper in stem cuttings of the terrestrial medicinal plant, Portulaca oleracea Linn. Environmental Monitoring and Assessment 121(1-3): 233-244. https://doi.org/10. 1007/s10661-005-9117-1

Muthukumar T, Sarah J \& Dinesh-Babu S. 2018. Zinc influences regeneration of Talinum portulacifolium stem cuttings in nutrient solution. Notulae Scientia Biologicae 10(4): 530-539. https://doi.org/10.15835/ nsb10410 392

Nair NC \& Henry AN. 1983. Flora of Tamil Nadu, India (Ser. 1). India: Botanical Survey of India.

Nessa F, Khan SA \& Abu Shawish KY. 2016. Lead, cadmium and nickel contents of some medicinal agents. Indian Journal of Pharmaceutical Science 78(1):111119. http://dx.doi.org/10.4103/0250-474X.180260

Niu ZX, Sun LN, Sun TH \& Li YS, Hong W. 2007. Evaluation of phytoextracting cadmium and lead by sunflower, ricinus, alfalfa and mustard in hydroponic culture. Journal of Environmental Sciences 19(8): 961967. https://doi.org/10.1016/S1001-0742(07)60158-2

Obasi NA, Akubugwo El, Kalu KM \& Ugbogu OC. 2013. Speciation of heavy metals and phyto-accumulation potentials of selected plants on major dumpsites in Umuahia, Abia State, Nigeria. International Journal of Current Biochemistry Research 1(4): 16-28.

Obasi NA, Obasi SE, Akubugwo El, Elom SO \& Alisa CO. 2009. Health risk assessment of selected wild valuable plants species grown around Amaozara and Amaechara aged dumpsite soils in Amasiri, Afikpo North LGA of Ebonyi State, Nigeria. 3rd International Conference on Chemical, Agricultural and Medical Sciences (CAMS-2015) Dec. 10-11, 2015 Singapore, pp 23-27. http://dx.doi.org/10.15242/ IICBE.C1215029

Obasi NA, Obasi SE, Igbolekwu RI, Nkama OJ \& Eluu SO. 2015. Health risk assessment of the quality of plants cultivated around Eluogo and Agboogo Ibii aged dumpsites in Ibii, Afikpo North LGA of Ebonyi State, Nigeria. American Journal of Environmental Engineering Science 2(6): 100-108.

Piper CS. 1966. Soil and plant analysis. Bombay, India: Hans Publishers.

Pollard AJ, Powell KD, Harper FA \& Smith JAC. 2002. The genetic basis of metal hyperaccumulation in plants. Critical Reviews in Plant Science 21(6): 539566. https://doi.org/10.1080/0735-260291044359

Rajkumar K, Sivakumar S, Senthilkumar P, Prabha D, Subbhuraam CV \& Song YC. 2009. Effects of selected heavy metals $(\mathrm{Pb}, \mathrm{Cu}, \mathrm{Ni}$ and $\mathrm{Cd})$ in the aquatic medium on the restoration potential and accumulation in the stem cuttings of the terrestrial plant, Talinum triangulare Linn. Ecotoxicology 18(7): 952960. https://doi.org/10.1007/s10646-009-0371-9

Ronzan M, Piacentini D, Fattorini L, Della-Rovere F, Eiche E, Riemann M, . . . Falasca G. 2018. Cadmium and arsenic affect root development in Oryza sativa L. negatively interacting with auxin. Environmental and Experimental Botany 151: 64-75. https:// doi.org/10.1016/j.envexpbot.2018.04.008

Sankara-Rao K, Swamy RK, Kumar D, Arun-Singh R \&
Gopalakrishna-Bhat K. 2019. Flora of Peninsular India. Available in http://flora-peninsula-indica.ces.iisc. ac.in/herbsheet.php?id=8108\&cat $=7$ (Accessed 29 April 2019).

Santos KM \& Fisher PR \& Argo WR. 2009. Stem versus foliar uptake during propagation of Petuniax hybrida vegetative cuttings. HortScience 44(7): 1974-1977. https://doi.org/10.21273/HORTSCI.44.7.1974

Sekhar CK, Kamala CT, Chary NS \& Mukherjee AB. 2007. Arsenic accumulation by Talinum cuneifoliumapplication for phytoremediation of arsenic contaminated soils of Patancheru, Hyderabad, India. Trace Metal and other Contaminants in the Environment 9: 315-338. https://doi.org/10.1016/S1875-1121(06) 09 $\underline{012-2}$

Shahid M, Dumat C, Khalid S, Niazi NK \& Antunes PMC. 2016. Cadmium bioavailability, uptake, toxicity and detoxification in soil-plant system. Reviews of Environmental Contamination and Toxicology 241: 73-137. https://doi.org/10.1007/398_2016_8

Steffens B \& Rasmussen A. 2016. The physiology of adventitious roots. Plant Physiology 170(2): 603-617. https://doi.org/10.1104/pp.15.01360

Sun S, Li M, Zuo J, Jiang W, Liu D. 2015. Cadmium effects on mineral accumulation, antioxidant defence system and gas exchange in cucumber. Zemdirbyste-Agriculture 102(2): 193-200. https://doi.org/ 10.13080/z-a.2015.102.025

Swarna J, Ravindhran R \& Lokeswari TS. 2015. Characterization of Talinum triangulare (Jacq.) Willd. germplasm using molecular descriptors. South African Journal of Botany 97: 59-68. http://dx.doi.org/10. 1016/j.sajb.2014.12.012

Thangavel P, Subburam V. 1998. Effect of trace metals on the restoration potential of leaves of the medicinal plant, Portulaca oleracea Linn. Biological Trace Element Research 61(3): 313-321. https://doi.org/10. 1007/BF02789091

Thévenod F \& Lee WK. 2013. Toxicology of cadmium and its damage to mammalian organs. Metal lons in Life Sciences 11: 415-490. http://dx.doi.org/10.1007/978-94-007-5179-8 14

Vysotskaya L, Cherkozyanova A, Veselov S \& Kudoyarova G. 2007. Role of Auxins and cytokinins in the development of lateral roots in wheat plants with several roots removed. Russian Journal of Plant Physiology 54(3): 402-406. https://doi.org/10.1134/ S1021443707030168

Waisberg M, Joseph P, Hale B \& Beyersmann D. 2003. Molecular and cellular mechanisms of cadmium carcinogenesis. Toxicology 192(2-3): 95-117. https://doi. org/10.1016/S0300-483X(03)00305-6

Wang W, Wu Y, Akbar S, Jia X, He Z \& Tian X. 2016. Effect of heavy metals combined stress on growth and metals accumulation of three Salix species with different cutting position. International Journal of Phytoremediation 18(8): 761-767. https://doi.org/10. 1080/15226514.2015.1131237

Wilkins DA. 1978. The measurement of tolerance to edaphic factors by means of root growth. New Phytologist 136(3): 623-633. https://doi.org/10.1111/j.14698137.1978.tb01595.x 
Yao X, Ma F, Li Y, Ding X, Zou D, Niu Y, . . Deng J. 2018. Effect of water cadmium concentration and water level on the growth performance of Salix triandroides cuttings. Environmental Science and Pollution Research 25(8): 8002-8011. https://doi.org/ 10.1007/s11356-017-1158-9

Zacchini M, Pietrini F, Mugnozza GS, lori V, Pietrosanti L \& Massacci A. 2009. Metal tolerance, accumulation and translocation in poplar and willow clones treated with cadmium in hydroponics. Water, Air, and Soil Pollution 197(1): 23-34. https://doi.org/10.1007/s112 70-008-9788-7

Zha HG, Jiang RF, Zhao FJ, Vooijs R, Schat $H$, Barker HA \& McGrath HP. 2004. Co-segregation analysis of cadmium and zinc accumulation in Thlaspi caerulescens interecotypic crosses. New Phytologist 163(2): 299-312. https://doi.org/10.1111/j.1469-8137.2004. 01113.x 